\title{
Controlled trial of immune response of preterm infants to recombinant hepatitis B and inactivated poliovirus vaccines administered simultaneously shortly after birth
}

Nehama Linder, Rachel Handsher, Boris German, Lea Sirota, Mike Bachman, Sigal Zinger, Ella Mendelson, Asher Barzilai

\begin{abstract}
Aim-The study was conducted to evaluate the immunogenicity of an early, extra dose of enhanced inactivated poliovirus vaccine (IPV) administered simultaneously with recombinant hepatitis $B$ vaccine $(\mathrm{HBV})$ to preterm infants shortly after birth.
\end{abstract}

Methods-Three groups were studied. Fifty preterm infants received IPV intramuscularly within 24 hours of birth, in addition to routine recommended childhood immunisations. Fifty two preterm infants and 35 full term infants received routine immunisations only (routine vaccination timing: HBV at birth, 1 and 6 months of age; IPV at 2 and 4 months; oral polio vaccine (OPV) at 4 and 6 months; diphtheria-tetanus-pertussis (DTP) at 2, 4, and 6 months; and Haemophilus influenzae $B$ vaccine at 2 and 4 months). Blood samples were taken at birth, 3 and 7 months of age from all infants, and at 1 month of age from preterm infants only.

Results-At birth, a lower percentage of both study and control preterm infants had antipoliovirus type 3 titres $\geqslant 1: 8$ than full term infants. At 1 and 3 months of age significantly more early IPV infants had antipoliovirus type 3 titres $\geqslant 1: 8$ than routinely vaccinated preterm infants $(p<0.05)$. At 7 months of age there were no significant differences in percentage of antipoliovirus titres $\geqslant 1: 8$ or geometric mean times (GMTs) between the early IPV group and the routinely vaccinated preterm group. At 3 and 7 months of age, the percentage of positive antihepatitis $B$ titres $(\geqslant 1: 10)$ and the GMT of the early IPV preterm group did not differ significantly from those of preterm controls. There was no significant difference in percentage of positive antihepatitis $B$ titres between the early IPV group and full term controls at any time. GMTs for hepatitis B antibodies were significantly lower in the early IPV preterm group than in full term controls at 3 and 7 months of age.

Conclusions-Administration of an additional dose of IPV simultaneously with routine $\mathrm{HBV}$ to preterm infants shortly after birth provides early protection from poliovirus and hepatitis $B$ infection, and does not interfere with poliovirus antibody production at the age of 7 months.

(Arch Dis Child Fetal Neonatal Ed 2000;83:F24-F27)
Keywords: preterm infants; hepatitis B; poliovirus; vaccination; antibody

During the summer of 1988, an outbreak of poliovirus type 1 occurred in Israel. One of the victims was a 2 month old baby who had not yet been immunised. ${ }^{1}$ Two studies performed since then have shown that approximately $50 \%$ of preterm infants lack positive titres $(\geqslant 1: 8)$ to at least one of the poliovirus types, and may benefit from early vaccination with inactivated polio vaccine (IPV). ${ }^{23}$

In 1992 hepatitis $B$ vaccine (HBV) was introduced in Israel for vaccination of all newborns, including preterm infants, soon after birth. Concomitant administration of HBV and IPV has been shown to be effective in full term infants, ${ }^{4}$ but there are no studies in preterm infants.

This study aimed to evaluate the immunogenicity of IPV and HBV administered simultaneously to premature infants within the first 24 hours of life.

\section{Materials and methods}

SUBJECTS

The study population included 177 infants: 127 preterm infants and 50 full term infants. Preterm infants were born between June and December 1994 (gestational age 30-35 weeks, weight $>1000 \mathrm{~g})$. Fifty healthy full term infants (gestational age $>37$ weeks, weight $>2500 \mathrm{~g}$ ), born consecutively in the morning hours between 1 June 1994 and 15 June 1994 were recruited as controls. Forty children were excluded: 22 preterm infants who received blood products, three preterm infants with sepsis, and 15 full term infants who were withdrawn by their parents after the first blood test.

The preterm infants were divided into a study group (group $\mathrm{A} ; \mathrm{n}=50$ ) and a control group (group $\mathrm{B} ; \mathrm{n}=52$ ) by $1: 1$ randomisation using a blinded envelope drawn by the parents. All full term infants were included in control group C.

The study protocol was approved by the hospital human ethics committee and informed parental consent was obtained for all subjects.

Infants in the study group A received an extra, early dose of IPV within 24 hours of birth, simultaneously with the routine HBV, followed by all routine vaccinations at the appropriate times (table 1). The early IPV was administered to one thigh and the HBV to the 
Table 1 Vaccination protocol: first six months of life

\begin{tabular}{llllll}
\hline Vaccination & Birth & One month & Two months & Four months & Six months \\
\hline IPV & $\mathrm{X}^{\star}$ & & $\mathrm{X}$ & $\mathrm{X}$ & $\mathrm{X}$ \\
OPV & $\mathrm{X}$ & $\mathrm{X}$ & & $\mathrm{X}$ & $\mathrm{X}$ \\
HBV & & & $\mathrm{X}$ & $\mathrm{X}$ & $\mathrm{X}$ \\
DTP & & $\mathrm{X}$ & $\mathrm{X}$ & \\
HiB & & & & \\
\hline
\end{tabular}

${ }^{\star}$ Only study group A received IPV at birth.

IPV, enhanced inactivated poliovirus vaccine, one ampoule intramuscularly (RIVM, Bilthoven, Holland); OPV, oral poliovirus vaccine, two drops by mouth (SmithKline Beecham, Rixemsart, Belgium); HBV, hepatitis B vaccine, $0.5 \mathrm{ml}(10 \mu \mathrm{g} / 0.5 \mathrm{ml})$ intramuscularly (Engerix; SmithKline Beecham, Belgium); DTP, diphtheria-tetanus-pertussis vaccine, $0.5 \mathrm{ml}$ intramuscularly (Pasteu Merieux, Lyon, France); HiB, Haemophilus influenzae B vaccine intramuscularly (H-B-VAX; Merck, Sharp and Dohme, West Point, USA).

Table 2 Clinical characteristics

\begin{tabular}{llll}
\hline & $\begin{array}{l}\text { Preterm study } \\
\text { group }(A) \\
(n=50)\end{array}$ & $\begin{array}{l}\text { Preterm control } \\
\text { group }(B) \\
(n=52)\end{array}$ & $\begin{array}{l}\text { Full term control } \\
\text { group }(C) \\
(n=35)\end{array}$ \\
\hline $\begin{array}{l}\text { Male/female } \\
\text { Median (range) gestational age (weeks) }\end{array}$ & $23 / 27$ & $22 / 30$ & $17 / 18$ \\
Mean (SD) birth weight (g) & $1528(384)$ & $32(30-35)$ & $40(38-42)$ \\
\hline
\end{tabular}

other thigh. Preterm infants in group B and full term group $\mathrm{C}$ received only routine immunisations (table 1).

Blood samples for the determination of antibody titres were obtained from all infants within the first 24 hours of life and at 3 and 7 months of age, and additionally from infants in groups $\mathrm{A}$ and $\mathrm{B}$ at 1 month of age.

SEROLOGY

Sera were kept frozen at $-20^{\circ} \mathrm{C}$ until tested using a microneutralisation system for the three poliovirus antibody serotypes. ${ }^{5}$ Serial twofold dilutions of sera were prepared and divided into aliquots in duplicate into 96-well microtitre plates; $32-100 \mathrm{TCID}_{50}$ of poliovirus were added to each well. The mixtures were incubated at $36^{\circ} \mathrm{C}$ for 20 hours, and then $20000 \mathrm{Hep}-2$ cells were added to each well. The plates were incubated at $36^{\circ} \mathrm{C}$ for four days. On the fifth day, cells were fixed, stained, and examined macroscopically for cytopathic effects.

Titres were determined as the highest dilution of serum protecting $50 \%$ of the cultures against $32-100 \mathrm{TCID}_{50}$ of challenge virus. Titres $\geqslant 1: 8$ were considered seropositive. ${ }^{6}$

Hepatitis B surface antibodies (HBsAB) were tested for by radioimmunoassay and interpreted as instructed by the manufacturer (Abbott Diagnostics, Chicago, Illinois, USA). A positive $\mathrm{HBs} A B$ titre was defined as $\geqslant 1: 10$ $\mathrm{miu} / \mathrm{ml}$.

STATISTICAL ANALYSIS

Statistical analysis was performed using the BMDP Statistical Software Package. ${ }^{7}$ The pro-

Table 3 Percentage of infants with protective antibody titres $\geqslant 1: 8$ for the poliovirus types 1, 2, and 3

\begin{tabular}{|c|c|c|c|c|c|c|c|c|c|}
\hline \multirow[b]{2}{*}{ Age } & \multicolumn{3}{|c|}{ Poliovirus type 1} & \multicolumn{3}{|c|}{ Poliovirus type 2} & \multicolumn{3}{|c|}{ Poliovirus type 3} \\
\hline & $A$ & $B$ & $C$ & $A$ & $B$ & $C$ & $A$ & $B$ & $C$ \\
\hline 24 hours & 100 & 98 & 100 & 100 & 100 & 100 & $90 \ddagger$ & $88 \dagger$ & 97 \\
\hline 1 month & 100 & 94 & ND & 100 & 98 & ND & $94^{\star}$ & 81 & ND \\
\hline 3 months & 100 & 98 & 100 & 100 & 98 & 100 & $98^{\star}$ & $85 \dagger$ & 100 \\
\hline 7 months & 98 & 100 & 100 & 100 & 100 & 100 & 100 & 98 & 100 \\
\hline
\end{tabular}

${ }^{\star} \mathrm{p}<0.05(\mathrm{~A} v \mathrm{~B}) ; \mathrm{tp}<0.05(\mathrm{~B} v \mathrm{C}) ; \ddagger \mathrm{p}<0.05$ (A $\left.v \mathrm{C}\right)$; all significance values follow Bonferroni’s correction for multiple comparisons.

A, group A ( $=50) ; B$, group B $(n=52)$; , group C $(n=35) ; N D$, not done.

portion of infants in each group with detectable antibodies to poliovirus types 1, 2, and 3 was compared for each type of antibody using the Pearson $\chi^{2}$ test. Seroconversion was defined as the appearance of neutralising antibodies in seronegative infants or a fourfold or greater increase over the titre expected following the decay of transplacentally acquired antibodies. The expected titre was calculated using an antibody half life of 28 days. ${ }^{89}$ The proportion of infants seroconverting was compared for each type of antibody using the Pearson $\chi^{2}$ test.

The geometric mean titres (GMTs) for antipolio were derived from the mean values after $\log _{2}$ transformations. Titres $<1$ :8 were calculated as 1:4, whereas titres $>1: 8192$ were calculated as 1:16 384. The GMTs for antihepatitis B were calculated from the $\log _{10}$ of the titres. Titres $<1: 10$ were calculated as 1:5, while titres $>1: 1000$ were calculated as 1:2000.

To determine the significance of the differences in antibody levels, we applied analysis of variance to the $\log _{2}$ values (antipolio titres) and the $\log _{10}$ values (antihepatitis B titres) of the reciprocal antibody titres with Bonferroni's correction for multiple comparisons. All statistical tests were two sided with a significance level of $\mathrm{p}<0.05$.

\section{Results}

Patient characteristics are presented in table 2. Groups A and B were similar for sex distribution, median gestational age, and mean birth weight. No local or systemic side effects to either vaccine were noted.

POLIO

The percentage of infants with antibody titres $\geqslant 1: 8$ and the GMTs for poliovirus types 1,2 , and 3 are shown in tables 3 and 4 .

At birth, a lower percentage of preterm infants (from both preterm groups) than full term infants had antipoliovirus type 3 antibody titres $\geqslant 1: 8(p<0.05)$. A significantly higher number of early IPV group A premature infants had antipoliovirus type 3 titres $\geqslant 1: 8$ than routinely vaccinated premature controls (group B) at 1 and 3 months $(\mathrm{p}<0.05)$. At age 3 months, group A had significantly higher GMT levels for poliovirus 2 and 3 than group B (317.4 $v$ 106.2; p < 0.01 and $218.3 v 72.0$; $\mathrm{p}<0.05$ respectively). At 7 months there were no differences in percentage of infants with positive antibody titres $\geqslant 1: 8$ or GMT levels among the three groups.

Lower antibody titres $(<1: 128)$ at birth to each of the three poliovirus types were 
Table 4 Geometric mean titres for antibodies to poliovirus type 1, 2, and 3

\begin{tabular}{|c|c|c|c|c|c|c|c|c|c|}
\hline \multirow[b]{2}{*}{ Age } & \multicolumn{3}{|c|}{ Poliovirus type 1} & \multicolumn{3}{|c|}{ Poliovirus type 2} & \multicolumn{3}{|c|}{ Poliovirus type 3} \\
\hline & $A$ & $B$ & $C$ & $A$ & $B$ & $C$ & $A$ & $B$ & $C$ \\
\hline 24 hours & $189 \ddagger$ & $163 \dagger$ & 534 & 402 & $288 \dagger$ & 6766 & 39 & $32 \dagger$ & 72 \\
\hline 1 month & 117 & 79 & ND & 214 & 153 & ND & 38 & 23 & ND \\
\hline 3 months & 98 & 81 & 159 & $317^{\star}$ & 106 & 167 & $218^{\star}$ & 72 & 87 \\
\hline 7 months & 1144 & 1121 & 1783 & 5078 & 3875 & 4905 & 1479 & 1499 & 1479 \\
\hline
\end{tabular}

${ }^{*} \mathrm{p}<0.05(\mathrm{~A} v \mathrm{~B}) ; \mathrm{tp}<0.05(\mathrm{~B} v \mathrm{C}) ; \neq \mathrm{p}<0.05$ (A $v$ C); all significance values follow Bonferroni's correction for multiple comparisons.

A, group A $(n=50)$; B, group B $(n=52)$; , group C $(n=35)$; ND, not done.

Table 5 Geometric mean titres (GMT) for hepatitis B surface antibody and percentage of infants with titres $\geqslant 1: 10$

\begin{tabular}{llllllll}
\hline & \multicolumn{3}{c}{ GMTs for hepatitis $B$} & & & \multicolumn{3}{c}{$\%$ with antibody titres $\geqslant 1: 10$} \\
\cline { 2 - 3 } \cline { 7 - 8 } Age & $A$ & $B$ & $C$ & & $A$ & $B$ & $C$ \\
\hline 24 hours & 0 & 0 & 0 & & 0 & 0 & 0 \\
1 month & $21^{\star}$ & 10 & ND & & 30 & 4 & ND \\
3 months & $91 \ddagger$ & $51 \dagger$ & 185 & & 74 & $65 \dagger$ & 86 \\
7 months & $273 \ddagger$ & 420 & 653 & & 92 & $79 \dagger$ & 94 \\
\hline
\end{tabular}

${ }^{\star} \mathrm{p}<0.05(\mathrm{~A} v \mathrm{~B}) ; \mathrm{tp}<0.05(\mathrm{~B} v \mathrm{C}) ; \neq \mathrm{p}<0.05(\mathrm{~A} v \mathrm{C})$; all significance values follow

Bonferroni's correction for multiple comparisons.

A, group A $(n=50) ; B$, group B $(n=52) ; C$, group C $(n=35) ; N D$, not done.

associated with a better serological response in all three groups one month after IPV immunisation $\left(\mathrm{p}<0.01\right.$, Pearson $\chi^{2}$ test). In early IPV group $\mathrm{A}$, seroconversion at 1 month of age occurred in $5.4 \%, 13.5 \%$, and $35.1 \%$ of patients for antibodies to poliovirus types 1,2, and 3 , respectively. At age 3 months, the only significant difference between groups was between study group A and the full term control group C; $52.8 \%$ and $20 \%$ respectively seroconverted for anti-poliovirus $2(\mathrm{p}<0.03)$. For poliovirus types 1 and 3 no seroconversion differences between any two groups were noted. There were no differences between groups at 7 months.

HEPATITIS B

Table 5 shows the percentage of infants with antibody titres $\geqslant 1: 10$ and the GMTs for antihepatitis B. There was no significant difference between the proportion of early IPV infants reaching positive antihepatitis B titres in either of the control groups at 3 and 7 months of age. There was no difference in antihepatitis B GMT between group A and group B at 3 and 7 months of age. However, the early IPV (group A) GMT was significantly lower than that of full term controls at 3 and 7 months of age.

\section{Discussion}

During recent outbreaks of poliomyelitis in Israe ${ }^{1}$ and the Netherlands, ${ }^{10}$ several infants who had not yet received their first routine immunisation were affected. In developing countries where poliomyelitis and hepatitis B are endemic, infection with both viruses can occur simultaneously. Early, conjoint administration of viral vaccinations would be advantageous for preterm infants who are a particularly vulnerable group because of lower titres of maternally acquired antibodies. ${ }^{11}$ Good immune response to oral polio vaccine (OPV) and IPV given to preterm infants at two months of age has been reported. ${ }^{12}{ }^{13}$ Pagano et al were the first to report a good response of preterm infants to immunisation against poliovirus 1 with OPV given soon after birth. ${ }^{14}$ Our group found previously that administration of IPV to preterm infants soon after birth significantly decreases the proportion of infants susceptible to poliovirus type 3, and raises the GMTs for poliovirus types 1 and 3 at the age of 3 months. ${ }^{2}$ Although the age when hepatitis $B$ vaccination for preterm infants should be initiated is controversial, it is important that it is administered early to preterm infants living in countries where the disease is endemic. ${ }^{15-19}$

The results of the present study confirm that immunisation of preterm infants with IPV and HBV soon after birth results in a significantly higher proportion of infants with positive antibody titres against poliovirus 3 at 1 and 3 months of age. In addition, early immunisation resulted in significantly higher GMT levels for antipoliovirus types 2 and 3 at age 3 months

One of the problems associated with early immunisation is the possible interference of maternal antibodies in the infant's immune response. Dong et al showed that the seroconversion is delayed in the presence of high maternal antibodies. ${ }^{20}$ This is true for poliovirus vaccine administered to full term infants during the first 24 hours of life. ${ }^{21}$ In this study, lower antibody titres at birth were associated with a better immune response to all the poliovirus types in both the study and the control groups.

Concurrent administration of HBV and IPV has been studied in full term infants. No impairment in the expected protective efficacy was noted when HBV was administered with: BCG and IPV, ${ }^{4}$ diphtheria-tetanus (DT) and diphtheria-tetanus-pertussis (DTP), ${ }^{22} \mathrm{OPV},{ }^{22-24}$ group A meningococcus vaccine, ${ }^{25}$ measles vaccine ${ }^{26}$ Japanese $\mathrm{B}$ encephalitis vaccine,${ }^{26}$ and hepatitis A vaccine..$^{27}{ }^{28}$ Our study is the first to investigate early simultaneous HBV and IPV vaccination in preterm infants. The percentage of early IPV infants who reached protective antihepatitis B titres by 7 months of age was similar to full term controls, although the study group antihepatitis B GMT was significantly lower. The cause is unclear and is most likely the effect of gestational age.

We conclude that the addition of an early IPV to the vaccination protocol significantly decreases the proportion of preterm infants susceptible to poliovirus type 3 at 1 and 3 months of age. Simultaneous administration of HBV and IPV to preterm infants within 24 hours of birth appears to be safe and effective, providing early protection against the two diseases. This approach may be useful in countries where both hepatitis B and poliomyelitis are endemic.

This study was supported by the Chief Scientist of the Israel Ministry of Health. The authors are grateful to Mrs Pearl Lilos of the Department of Statistics, Tel Aviv University for performing the data analysis, and to Mrs M Neuman and Mrs B Abramowitz of the Central Virology Laboratory for their excellent technical assistance.

1 Slater PE, Orenstein WA, Morag A, et al. Poliomyelitis outbreak in Israel in 1988: a report with two commentaries. Lancet 1990;335:1192-5. 
2 Linder N, Yaron M, Handsher R, et al. Early immunization with inactivated poliovirus vaccine in premature infants. $\mathcal{F}$ with inactivated poliovirus

3 Linder N, Taushtein I, Handsher R, et al. Placental transfer of poliovirus antibodies in preterm infants. Vaccine 1998;16:236-9.

4 Coursaget P, Relyveld, E, Brizard A, et al. Simultaneous injection of hepatitis B vaccine with BCG and killed poliovirus vaccine. Vaccine 1992;10:319-21

5 Albrecht P, Van Steenis G, Van Wezel AC, Sack J. Standardization of poliovirus neutralizing antibody tests. Rev Infect Dis 1984;6(suppl 2):540.

$6 \mathrm{WHO} / \mathrm{EPI} / \mathrm{CDS} / \mathrm{POLIO} / 90.1$. Manual for the virological investigation of poliomyelitis. Geneva: World Health Organization, 1990:95.

7 Dixon WJ, chief ed. BMDP - Statistical Software. Berkeley: California: University of California Press, 1990

8 Weckx LY, Schmidt BJ, Herrmann AA, Miyasaki CH, Novo NF. Early immunization of neonates with trivalent oral NF. Early immunization of neonates with
polio vaccine. Bull WHO 1992;70:85-91.

9 Halsey N, Galazka A. The efficacy of DPT and oral poliomyelitis immunization schedules initiated from birth poliomyelitis immunization schedules initiated from
to 12 weeks of age. Bull WHO 1985;63:1151-69.

10 World Health Organization. Surveillance of poliomyelitis: lessons learnt from the 1992/1993 outbreak in The Netherlands. In: Report on a World Health Organization Meeting, Bilthoven, Holland. Geneva: World Health Organization, 1994

11 Evans HE, Akpata SO, Glass L. Serum immunoglobulin levels in preterm and full term infants. Am f Clin Patho 1971;560:416-18.

12 Smolen P, Bland R, Heiligenstein E, et al. Antibody response to oral polio vaccine in premature infants. $f$ Pediat 1983;103:917-19.

13 Adenyi-Jones SC, Faden H, Ferdon MB, Kwong MS, Ogra PL. Systemic and local immune responses to enhancedpotency inactivated poliovirus vaccine in premature and potency inactivated poliovirus vaccine
term infants. $\mathcal{F}$ Pediatr 1992;120:686-9.

14 Pagano JS, Cornely D, Plotkin SA. The response of preterm infants to infection with attenuated poliovirus. Pediatrics infants to infection

15 Chawareewong S, Jirapongsa A, Lokaphadhana K. Immune response to hepatitis $\mathrm{B}$ vaccination and preterm neonates. Southeast Asian f Trop Med Public Health 1991;22:39-40. 16 Lau YL, Tam AY, Ng KW, et al. Clinical and laboratory
observations: responses of preterm infants to hepatitis B vaccine. F Pediatr 1992;121:962-5.

17 Committee on Infectious Diseases, American Academy of preterm infants and for children with lapsed immunization. Pediatrics 1994:94:403-4.

18 Kim SC, Chung EK, Hodinka RL, et al. Immunogenicity of hepatitis B vaccine in preterm infants. Pediatrics 1997;99:534-6

19 Blondheim O, Bader D, Abend M, et al. Immunogenicity of hepatitis B vaccine in pre-term infants. Arch Dis Child 1998;79:206-8.

20 Dong-DX, Hu XM, Liu WJ, et al. Immunization of neonates with trivalent oral poliomyelitis vaccine (Sabin). Bull WHO 1986;64:853-60.

21 Swartz TA, Handsher R, Stoeckel P, et al. Immunologic memory induced at birth by immunization with inactimemory induced at birth by immunization with inactivated polio vaccin $1989 ; 5: 143-5$.

22 Yuan C. Immune response and post inoculation reactions of simultaneous administration of hepatitis B vaccine with routine vaccine in children, I. Immune response and post inoculation reactions of simultaneous administration of DPT, TOPV and Hepatitis B vaccine. Chung Hau Liu Hsing Ping Hsueh Tsa Chin 1989;10:206-9.

23 Barone P, Mauro L, Leonardi S. Simultaneous administration of HB recombinant vaccine with diphtheria and tetanus toxoid and oral polio vaccine: a pilot study. Acta Pediatr fpn 1991;33:455-8.

24 Giammanco G, Li-Volti S, Mauro L, et al. Immune response to simultaneous administration of a recombinant DNA hepatitis B vaccine and multiple compulsory vaccines in infancy. Vaccine 1991;9:747-50.

25 Yuan C. Immune response and post inoculation reactions of simultaneous administration of hepatitis B vaccine with routine vaccine in children, III. Immune response and post inoculation reactions of simultaneous administration of hepatitis B vaccine, BCG and meningococcus group A polysaccharide vaccine. Chung Hau Liu Hsing Ping Hsueh Tsa Chin 1990;11:34-7.

26 Yuan C. Immune response and post inoculation reactions of simultaneous administration of hepatitis B vaccine with routine vaccine in children. II. Immune response and post
inoculation reactions of simultaneous administration of hepatitis B vaccine with Japanese B encephalitis vaccine and measles vaccine. Chung Hau Liu Hsing Ping Hsueh Tsa Chin 1989;10:210-14.

27 Ambrosch F, Andre FE, Delem A, et al. Simultaneous vaccination against hepatitis A and B: results of a controlled study. Vaccine 1992;10(suppl 1):S142-5.

28 Flehming B, Heinricy V, Pfisterer M. Simultaneous vaccination for hepatitis A and B. F Infect Dis 1990;161:865-8. 